\title{
Risk Based Management Methods for Maintaining 750 kV Electricity Grid in Northwest China
}

\author{
Li Juan, Liang Naifeng, Zhao Puzhi, Zhou Erbiao, Li Xiaoguang
}

State Grid Xinjiang Electric Power Compangy, Xinjiang, China

Email address:

773963700@qq.com (Liang Naifeng), 13738674523@163.com (Li Juan)

\section{To cite this article:}

Li Juan, Liang Naifeng, Zhao Puzhi, Zhou Erbiao, Li Xiaoguang. Risk Based Management Methods for Maintaining 750 kV Electricity Grid in Northwest China. Journal of Electrical and Electronic Engineering. Vol. 3, No. 6, 2015, pp. 198-201. doi: 10.11648/j.jeee.20150306.14

\begin{abstract}
This paper will discuss a different case - a $750 \mathrm{kV}$ transmission grid in China, in which the regulation rules have not been decided and the assets are operating in their early stage. The difficulty in technology and insufficiency of regulation decide the focus of this study: to select the method feasible in this business environment, rather than to develop complicated but unrealistic models.
\end{abstract}

Keywords: Transmission, Maintenance, Management

\section{Introduction}

Electricity transmission operators (TSO) in China remain their monopoly status. However, in a national policy made in 2015, the focus of its regulation changes from the end-user price to the transmission tariff. This means that a Chinese TSO is required to clarify its transmission costs, which brings challenges to its maintenance department.

Traditionally, maintenance departments of the TSO's focus on developing repair techniques and performing repair activities in order to guarantee a high reliability of the network. Nowadays, economic and environmental responsibilities have also been assigned to maintenance managers. They are expected to organize the maintenance activities optimally in such a way that the profits and performances of the TSO are maximized. This elevates maintenance management to a new level, namely "asset management".

Meanwhile, the $750 \mathrm{kV}$ transmission network, as the backbone of electricity grid in northwest China, started just within a decade and keeps expanding in a rapid speed in the 2010s. For example, according to the planning, the total length of its $750 \mathrm{kV}$ overhead line will increase for over $150 \%$. In such a rapid growth, maintenance activities should be optimized not only for saving costs, but also for allocating limited human power and maintenance equipment. Moreover, the desert, mountainous and glacial environment in this area has introduced a high technical complexity to its maintenance activities. These are important considerations of establishing risk-based maintenance.
This paper will firstly review existing methods for riskbased maintenance from the perspective of asset managers in Section 2. Then, in Section 3, proper methods will be identified for the preliminary stage of risk-based maintenance on the studied $750 \mathrm{kV}$ grid.

\section{General Models of Risk-Based Maintenance}

\subsection{The Trigger of Introducing Risk-Based Maintenance in the Electricity Transmission Sector}

In tens of countries worldwide, the liberalized electricity market launched in the 1980's has brought a complete change to the power delivery sector. During the liberalization processes, governments divested themselves from the utility services and established the trade systems of utility products. Consequently, the corporatized electricity sector is operated by multiple companies whose business areas are defined by the regulator. This means, in the case of an electricity TSO, the involvement in generation and trade is gradually reduced or forbidden in many countries [1].

Before the implementation of the above mentioned regulations, the utility sector was protected by its natural monopoly status. This enabled the system operators to adopt risk-free development strategies such as "expanding the network up to its technical limits", or "enhance the reliability and redundancy with all available budgets". After the market liberalization, an electricity transmission or distribution 
company can no longer afford such expensive development strategy, since it exposes the asset owner to commercial risks. Coincidently, the growing public concerns regarding the environment and sustainability have led to stricter restrictions on the existence and expansion of the power grid [2] and its subsystems including overhead lines [3], substations [4] and cables [5].

Physical asset management [6], or asset management (AM) in short, is a field of emerging importance in the utility sector. The managed objects are physical assets and asset systems. Distinguished from human, information, financial and intangible assets, physical assets are a subset of fixed assets. They serve as the backbone of the daily operation within a power grid. Maintenance is a collection of the most discussed activities within in the domain of AM. Historically, three major advancements have been achieved and applied in maintenance management [7].

The first advancement is the abandoning of "Corrective Maintenance" (CM). Maintenance was, originally and yet in many occasions, corrective. In CM, components are completely replaced after they fail. In contrast, "Preventive Maintenance" (PM) intends to recover a component from a faulty state through proper repair techniques. The purpose of PM is primarily to avoid major failures and consequent interruptions of routine operation. However, in many cases, the use of PM can lead to the extension of the service life of components.

The second advancement is the introduction of maintenance strategies. In current practice, a maintenance strategy is essentially a method to schedule individual PM tasks within an asset fleet. Proper scheduling controls repair costs significantly, because required resources such as spare parts, transportation services, work force or cash flow are usually lower in costs if arranged in advance rather than requested on site. A basic scheduling approach is ranking health condition indices estimated for individual components. If the index is derived from the usage history of each component, the maintenance strategy is called "Time-Based Maintenance" (TBM). If diagnostic information from inspections, tests and monitors are utilized to assess the index, the maintenance strategy is "Condition-Based Maintenance" (CBM).

The third advancement is the introduction of risk analysis. Generally speaking, risk is an approach to describe the potentiality of an incident such as failure of a certain component. In management, a risk is featured with its probability and consequence and rated with the expected value - the multiplying product of the probability and the consequence.

Maintenance scheduling can be achieved through risk analysis. Risk analysis on an individual component consists of estimating probability and consequences quantitatively. Firstly, the rough level of failure probability can be derived from the health condition index. Secondly, the failure consequence is measured in multiple items[8]. Traditionally in power grids, the network reliability, mainly measured by the customer minute loss of the blackout, is the only item. This is the Reliability-Centered Maintenance (RCM) strategy. Recently, additional aspects such as finance, safety and environment were added in response to regulations and stakeholder requirements. This extends RCM to a complete Risk-Based Maintenance strategy (RBM).

Risk analysis can be performed not only on components to implement a maintenance strategy, but also on asset systems to guide and select a maintenance strategy on its composing components. This technique is frequently referred as the failure mode, effect and criticality analysis (FMECA). In FMECA, the risk analysis is performed on a failure mode. A failure mode describes a function loss on asset systems of different scales, e.g. switchgear, bus, or complete substation. Failure modes are distinguished from each other according to their original faulty component and the physical degradation mechanism. After determining probability, consequence and risk analysis procedures, FMECA is capable to rank failure modes with their expected risk indices. Using this ranking, asset managers can adapt maintenance strategies to the failure mode it tackled. e.g. for a component with highly ranked failure modes, $\mathrm{CM}$ is abandoned, frequency of TBM is increased, proper diagnostic tools for CBM is introduced, etc.

RBM and FMECA represent the up-to-date advancement of AM in electricity transmission and distribution. This enables the utility company to optimize the allocation of the maintenance budget.

\subsection{Introducing Risk-Based Maintenance at the Tactical Level of Asset Management}

Asset managers have widely accepted the triple level model as introduced in Table I. Specifically, for maintenance management, the condition indicator system is mainly implemented at the operational level to schedule maintenance plans, as the second advancement in II.A has stated. Meanwhile, the FMECA is implemented at the tactical level to decide maintenance methods and diagnostic methods, i.e. the maintenance strategies. This is mainly included in the third advancement in 2.1 .

Table 1. Features of the three levels of asset management (adapted from [8]).

\begin{tabular}{llllll}
\hline Level & Time frame & Concerned asset group & Performance & Expenditure \\
\hline Strategic & Long-term: $>$ a decade & Asset portfolio & Societal, Economic & Capital & Decision maker \\
Tactical & Mid-term: $3-7$ fiscal years & Asset system & Economic, Technical & Budget \\
Operational & Short-term: $<1$ fiscal year & Asset (item) & Technical & Cost & Asset manager \\
\hline
\end{tabular}

Currently, most advancements in maintenance management concentrate on developing diagnostic tools and standard condition indicator systems. These are progresses at the operation level. Moreover, many TSO's have started to construct their risk-based AM regime, including $\mathrm{RBM}$ at the operation level and FMECA at the tactical level. Extensive 
activities, including life cycle optimization at the operational level and investment decisions at the tactical level have been gradually introduced in specific cases to manage asset systems such as medium voltage cables, overhead lines and power transformers.

\section{Selecting Appropriate Methods for Risk-Based Management on 750 kV Assets}

\subsection{Features of the Studied $750 \mathrm{kV}$ Grid}

The studied $750 \mathrm{kV}$ have three features. They will decide our selection of risk assessment method in this section.

- Infant mortality. The studied assets are relatively new and rapidly increasing in population.

- Missing performance benchmarking. The ongoing changes on TSO regulation have not yet indicated any clear consequences on all incidents, especially regarding the environment and safety ones.

- Rich external knowledge on the failure modes, condition indicators of assets. The mother company of the TSO has nationally standardized testing and condition indicator system on major high-voltage assets. However, the effectivity of the standard indicators is undermined by the special operating environment in the wildness in deserts, mountains or glaciers.

Based on the above limitations, the studied risks yield to the failures of high-voltage components and consequent performance losses. At the tactical level of AM, this study should perform FMECA to adapt external knowledge on condition indicators to the specific operating environment of northwest China. The main benefit of such adaption will be the capability to: minimize the failure frequency of highvoltage components of the same type, through prioritizing the maintenance tasks on them.

\subsection{Estimation of the Probabilities of Failure Modes}

At the tactical level, the probably of a failure mode should be estimated, so that proper diagnostic method can be introduced as countermeasures. The capability to estimate of such probabilities is not automatically ready in a TSO.

A failure is defined as the situation where a certain object cannot function under stated conditions for a specified period of time. Within asset portfolios, a failure can occur at many different levels, such material, subcomponent, component/asset, asset system (including secondary equipment) and asset fleet/portfolio. When failures at a lower level lead to a failure at a higher level, the process is called a failure mechanism or failure mode.

The raw format of failure and life data is a list of failure events. A complete record on a failure event should include (1) the moment, (2) the (initially) failed object, (3) the cause or failure mechanism, (4) the reaction and countermeasures and (5) the consequences. [7]

From the raw format of failure records, two types of parameters can be derived as the conclusion after statistical analysis. They are the failure frequency, and the lifetime/time-to-failure (TTF). In order to convert time series of failure events to TTF, asset manager should record the population, namely the total number of investigated objects from which the failure events have been collected from. In addition, when the investigated objects are significantly different in their physical sizes, size information should also be included in population. (e.g. cables of different length). Such information comes from the installation/deployment data, which is typically the calendar date/time at which each object within the population started to operate/be stressed.

For the studied asset portfolio, the installation and population data is probably ready. However, since the assets are in their infant mortality stage, the TTF cannot be creditably estimated as in those aged asset populations. Therefore, the age-independent failure frequency is the main input to failure mode analysis.

\subsection{Risk Management: FMEA and ABC Classification Method}

In FMEA [9], as most standards have specified, the Risk Priority Number (RPN) is calculated for each failure mode. $\mathrm{RPN}$ is the product of the severity score, the probability score and the detectability score. As mentioned above, the probability score is the frequency of failure mode. The severity score represent the consequences of failure modes, which can be acquired from the external knowledge. The detectability score is not used in this preliminary stage.

$\mathrm{ABC}$ analysis method [10] is a method originally applied for quality control. It follows simple principles as follows. $20 \%$ products can have high values, which in total contain $70 \%$ value of the whole population. They form Class A which should be the focus of management. In our cases, the products are the failure modes, and the value is the RPN. The focus means that innovated condition diagnosis method, e.g. condition monitoring should be implemented. The next $30 \%$ products are of average values, i.e. in total contain $25 \%$ value of the whole population. They form Class B which should a certain degree of attention should be paid. In our case, such attention means standard diagnosis proposed by the national standards and external knowledge. The remaining $50 \%$ products are of low values, i.e. in total contain $5 \%$ value of the whole population. This means a minimally requested maintenance scheme.

The FMEA and ABC analysis is applicable when the performance benchmarking of managing electricity transmission assets (see examples in [11]) is still missing in China. The severity in FMEA and values in $\mathrm{ABC}$ analysis can be firstly narrowly defined as reliability and a few aspects of safety or environment, but later extended to other business values imposed by future laws.

\subsection{Continuous Improvements}

As time passes, the infant mortality of the studied asset portfolio will gradually stop. In this situation, the risk 
assessment proposed above can be improved in several aspects below:

- Optimize the condition indexing, namely the composing rules of condition indicators, for the special operating environment of northwest China.

- Specify different failure mechanisms on the same type of component in failure data, and treat them as different risks accordingly.

- Include detectability of diagnostic methods and preventability of repair methods in the RPN.

- Include not only the consequences on reliability, but also the consequences on safety and environment in the severity score of RPN.

\section{Conclusions}

Risk-based management on $750 \mathrm{kV}$ electricity transmission assets has been stimulated by the Chinese deregulation policy as well as the fast expansion of the asset population. When the assets are in their infant mortality and the definition on asset values are still missing, it is appropriate to balance costs and risks of failures, firstly through time-constant failure rate model, FMEA and $\mathrm{ABC}$ analysis method. These methods are compatible with future developments in aspects such as knowledge on assets, asset performance benchmarking, etc.

\section{References}

[1] Mohamed M Saied, Canceling the Power Frequency Magnetic and Electric Fields of Power Lines, IETE Journal of Education, 2013, pp.90-99.
[2] Strategic Environmental Assessment for Power Developments, Cigré Technical Brochure 487, 2012.

[3] Lin Che-Yun, Wang Alan X, Lee Beom Suk, Zhang Xingyu, Chen Ray T, High dynamic range electric field sensor for electromagnetic pulse detection, Optics Express, 2011, pp.17372-7.

[4] Improving the impact of existing substations on the environment, Cigré Technical Brochure 221, 2013.

[5] LIANG Cai, LIU Wenying, ZHOU Xichao, DAN Yangqing, Analysis on Role of $750 \mathrm{kV}$ Power Network Played in Loss Reduction of Gansu Power Grid, Power System Technology, 2012, pp.30-32.

[6] Specification for the optimized management of physical assets, British Standardisation Institute, 2008.

[7] Qikai Zhuang, Manaing Risks in Electrical Infrastructure Assets from a Strategic Perspective, Next Generation Infrastructure Foundations, Delft, 2015.

[8] E. Rijks, G. Sanchis, and P. Southwell, Asset Management Strategies for the 21st Century, ELECTRA, Cigré, vol. 248, 2010 .

[9] ZHOU Peipeng, XIANG Zutao, DU Ning, BAN Liangeng, XIE Guoping, Analyhsis on blocking of Qinghai-Tibet DC System Caused by Transformer Energizing in Northwest China 750 kv Grid, Automation of Electric Power Systems, 2013, pp.10-12.

[10] WANG Xiao-liang, DONG Hai-ying, REN Wei, State Evaluation of Secondary Device in $750 \mathrm{kV}$ Power Grid Based on Information Fusion, Proceedings of the Chinese Society of Universities for Electric Power System and its Automation, 2013, pp.24-26.

[11] Asset Management Performance Benchmarking, Cigré Technical Brochure 367, 2013. 